\title{
The effect of schizotypy and sex in social fear learning
}

Godoy-Giménez, M. ${ }^{1}$, González-Rodríguez, A. ${ }^{1}$, García-Pérez, A. ${ }^{1}$, Estévez, A. F. ${ }^{1,2}$, Cañadas, $F^{1,2}$ ${ }_{1}$ University of Almeria, Almeria, Spain

${ }^{2}$ CERNEP Research Centre, Almeria, Spain

\section{Background}

Schizotypy is a subclinical expression of symptoms qualitatively similar to those found in schizophrenia.

Some studies reflect abnormalities in fear conditioning in patients with schizophrenia (Holt et al., 2012). However, there are no studies exploring fear acquisition in individuals with schizotypy.

Therefore, the main aim of the present study is to explore if schizotypy influences the acquisition of fear through observation.

Besides, other studies suggest sex differences during fear conditioning experiments (Guimarães et al., 2013), so we will also explore the possible differential effect of schizotypy depending on sex.

\section{Participants}

Forty-eight psychology students (32 females, 16 males) from the University of Almeria.

\section{Materials}

- Schizotypal Personality Questionnaire (SPQ; Raine, 1991). Three factors: Cognitive-Perceptual, Interpersonal, and Disorganized.

NeXus-10 MKII (Mindmedia).

\section{Procedure}

- Learning phase never paired (see Figure 1). shock

\section{- Test phase} However, they never received a shock.
Our participants visualized a video of a person receiving electric shocks (US) most of the times a colour (CS+) was presented in a screen, while, a different colour (CS-), was

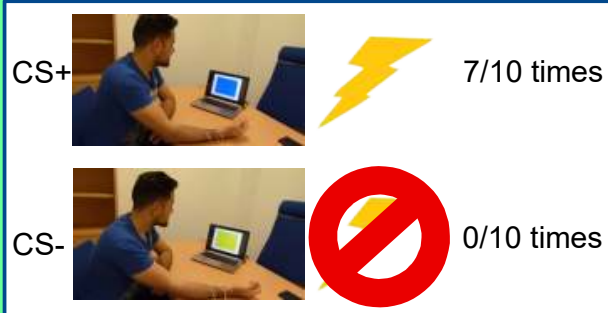

Figure 1. Example of the video. In this study, the CS+ (e.g., blue screen) was paired with a shock seven times (out of 10), and the CS(e.g., yellow screen) was never paired with a

Later on, participants were shown the same stimuli (in a different order), and they were told they will receive shocks paired with the same colour that was paired in the video. Skin Conductance Response (SCR) will be used as an index of fear conditioning learning (i.e., Olsson et al., 2007). These responses were corrected among participants as suggested in Lykken \& Venables (1971).

\section{Data analysis}

We analysed the SCRs of our participants in each phase of the experiment as a function of Sex and Stimulus using Welch-type statistics with trimmed means (10\%) and winsorized variances. Besides, we tested the effect of Schizotypy applying step-wise regression models separately for men and women, choosing as predictors the three factors of schizotypy, and as dependent variables, the SCRs when the first US -Learning- and the first CS+-Test- appeared.

\section{Results}

Omnibus contrasts revealed a significant effect of Stimulus in both Learning $\left(T_{\mathrm{WJ}}(1,24.8)=83, p<\right.$ $.001)$ and Test $\left(T_{\mathrm{WJ}}(1,28.2)=21.8, p<.001\right)$ phases. A significant effect of $\operatorname{Sex}\left(T_{\mathrm{WJ}}(1,23.9)=5, p=\right.$ .032 ) was also detected in the Learning phase. These results are shown in Fig 2.a and Fig 2.b.

Regarding Schizotypy, step-wise regression models revealed significant results only in the group of men. In the Learning phase, there were significant effects of the Interpersonal factor of Schizotypy in the SCRs of the First US $\left(\beta=-.59, R^{2}=.305, p=.016\right)$. In the Test phase, a significant effect of the Cognitive-Perceptual factor of Schizotypy was detected in the SCR of the First CS+ $\left(\beta=-.49, R^{2}=\right.$ $.196, p=.048)$. These results are shown in Fig 2.c and Fig 2.d a)

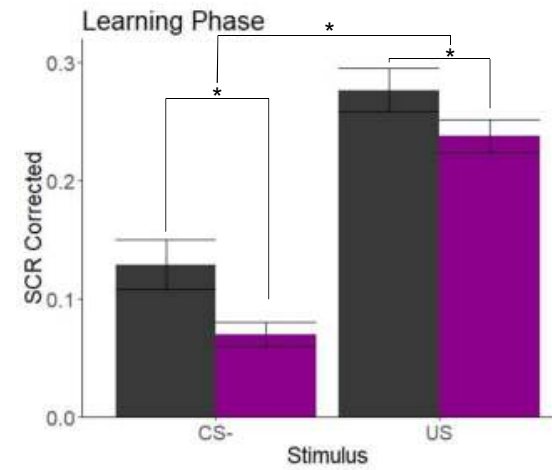

c) First US (Learning phase)

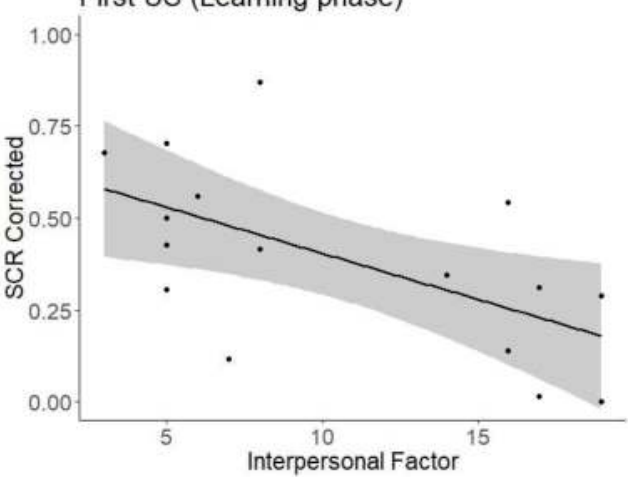

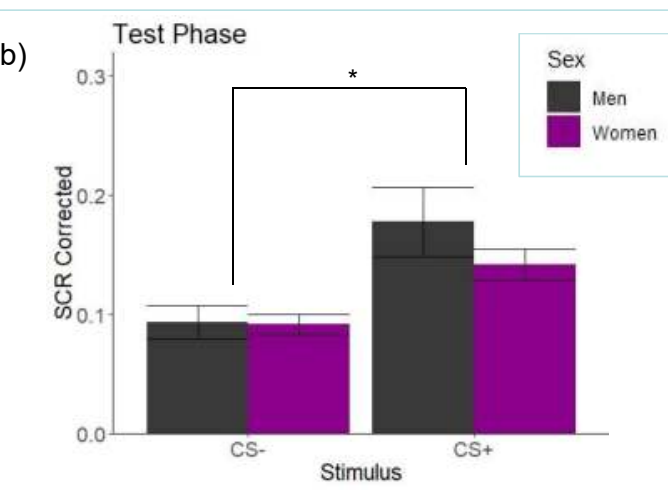

d)

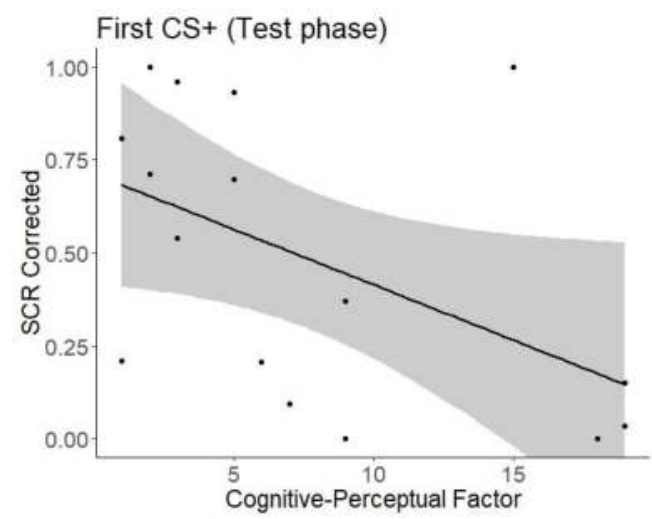

Figure 2. Graphic representation of regression analyses $(a, b)$ and mean comparisons $(c, d)$.

\section{Discussion}

The task seems to be working properly, since a CS+ always generated a larger SCR than a CSMen exhibited greater SCRs than women in the Learning phase for both the CS- and the US, suggesting a trend to a higher autonomic response towards any kind of stimulus. Furthermore, results suggest that some factors of schizotypy may influence social fear learning in men:

Interpersonal factor could be related with reduced empathy, since it seems to diminish the SCR of the participants when seeing other person receiving an electric shock.

Cognitive-Perceptual factor could be related with impaired fear conditioning, since, in the test phase, it seems to reduce the SCR when seeing a CS+. 